Itinéraires Itinéraires

Littérature, textes, cultures

2016-2 | 2017

Livre, sérialité et transmédialité

\title{
Performativité et éthique de la remédiation dans Dora Bruder, de Patrick Modiano
}

Performativity and The Ethics of Remediationin Patrick Modiano's Dora Bruder

\section{Élisabeth Routhier}

\section{OpenEdition}

Journals

Édition électronique

URL : http://journals.openedition.org/itineraires/3415

DOI : $10.4000 /$ itineraires.3415

ISSN : 2427-920X

Éditeur

Pléiade

Référence électronique

Élisabeth Routhier, «Performativité et éthique de la remédiation dans Dora Bruder, de Patrick Modiano », Itinéraires [En ligne], 2016-2 | 2017, mis en ligne le 25 avril 2017, consulté le 01 octobre 2020. URL : http://journals.openedition.org/itineraires/3415; DOI : https://doi.org/10.4000/itineraires.3415

Ce document a été généré automatiquement le 1 octobre 2020.

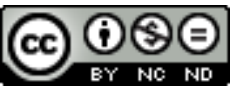

Itinéraires est mis à disposition selon les termes de la licence Creative Commons Attribution - Pas d'Utilisation Commerciale - Pas de Modification 4.0 International. 


\title{
Performativité et éthique de la remédiation dans Dora Bruder, de Patrick Modiano
}

\author{
Performativity and The Ethics of Remediationin Patrick Modiano's Dora Bruder
}

\author{
Élisabeth Routhier
}

\section{Introduction}

1 La genèse de Dora Bruder de Patrick Modiano (1997) ${ }^{1}$ est bien connue : en 1988, en feuilletant un journal datant de l'Occupation pour s'imprégner du climat et de l'ambiance de cette période - qui sert souvent de décor à son œuvre - l'auteur est frappé par l'avis de recherche d'une adolescente, Dora Bruder, dont il voit alors le nom pour la première fois. Modiano a raconté cette prémisse à quelques reprises après la parution du livre, mais elle fait également l'objet de précisions à l'intérieur même du texte. D'abord dans les toutes premières lignes, où l'avis de recherche est présenté entre guillemets, puis une quarantaine de pages plus loin, où l'avis initial, bien que présentant une forme légèrement modifiée, est intégré dans le flux du texte, sans les guillemets - c'est-à-dire plus près du geste d'énonciation et de la subjectivité de l'auteur :

En décembre 1988, après avoir lu l'avis de recherche de Dora Bruder dans le ParisSoir de décembre 1941, je n'ai cessé d'y penser durant des mois et des mois. L'extrême précision de quelques détails me hantait : 41 boulevard Ornano, 1m55, visage ovale, yeux gris-marron, manteau sport gris, pull-over bordeaux, jupe et chapeau bleu marine, chaussures sport marron. Et la nuit, l'inconnu, l'oubli, le néant tout autour. Il me semblait que je ne parviendrais jamais à retrouver la moindre trace de Dora Bruder. (DB : 53)

La fascination et le sentiment de vide éprouvés par Modiano à la suite de la lecture de cet avis de recherche ont, en réalité, engendré deux entreprises d'écriture différentes. De la première est issu un roman, Voyage de noces (1990), très librement inspiré de sa lecture du Paris-Soir, où une adolescente de l'âge de Dora Bruder, Ingrid Teyrsen, habite 
la rue Ornano et fait une fugue au début des années 1940. Modiano imagine une identité et une trajectoire qui auraient pu correspondre à celles de Dora, dont il ne connait encore presque rien, pas même la date et le lieu de naissance. La deuxième démarche d'écriture, celle de Dora Bruder, s'achève sept ans plus tard et entretient une relation beaucoup plus étroite avec la réalité : ce n'est plus, à proprement parler, une fiction. Le narrateur se confond avec l'auteur, brouillant ainsi le seuil entre ce qui pourrait constituer une diégèse et ce qui lui serait extérieur.

3 Il semble que les deux démarches correspondent en cela à deux mouvements distincts de réponse à la disparition, telle qu'elle est impliquée par l'avis de recherche de Dora Bruder. Si le «dispositif Voyage de noces - Dora Bruder» (Sheringham 2009) a quelques fois été étudié pour dégager les enjeux que l'un permet de mettre en évidence à propos de l'autre et vice versa, il semble que la relation entre l'écriture et la disparition en tant que telle est une piste qui reste à explorer. Les lieux, la nature et les effets de la disparition, ainsi que la position et les modes d'apparaître de Dora et d'Ingrid dans les modalités du disparaître, sont effectivement des aspects qui permettent de vérifier en quoi l'écriture de Dora Bruder se distingue des stratégies d'écriture qui traversent et caractérisent l'œuvre de Patrick Modiano.

\section{Le versant productif de la disparition}

4 Il faut d'abord rappeler que la disparition pose une série de problèmes essentiellement ontologiques. Un objet disparu est en fait un objet dont on postule une forme d'existence dans un ailleurs, mais dont la réalité effective ou empirique est incertaine. On en suppose tout à la fois la persistance ontologique et l'impossible contact immédiat, c'est-à-dire sans avoir recours à une forme de médiation. La disparition implique aussi une forme de prolongement ou une suite, sans quoi elle ne serait qu'une mort, une certitude, une fin qui ne poserait pas problème. Au contraire, il appert qu'elle a nécessairement une valeur productive, puisqu'elle convoque des nouvelles modalités d'existence de l'objet disparu, qui maintient ainsi une forme d'être au monde non identique. En effet, comme le souligne Santini, «la disparition est toujours un peu la condition d'apparition de quelque chose : lorsqu'une chose disparait, l'esprit, fasciné, tente d'en combler le vide sans jamais tout à fait y parvenir » (Santini 2007 : 35).

Si Santini fait ici référence à l'imaginaire et à la stimulation de l'esprit par le manque et le désir que provoque la disparition, je poursuivrais son idée en suggérant que ce qui émerge est une dynamique qui relève toujours-déjà de la (re)médiation. Le mouvement n'est donc pas linéaire ou téléologique, puisque c'est dans l'après-coup de la disparition que l'on reconnaît rétrospectivement que l'objet appartenait déjà au domaine de la médiation. Il suffit de penser au nom - et à l'acte même de nomination - pour comprendre cette idée. Que l'on traite des disparitions tragiques des régimes de terreur, de civilisations anéanties ou d'un simple objet égaré, nommer l'objet est le premier moyen dont on dispose pour lui assurer le maintien d'une part d'existence. Tant que le nom est enregistré et transmis, un être au monde minimal persiste. C'est aussi ce qui permet de définir et de circonscrire l'objet, de le convoquer dans un nouveau contexte, tout en rendant sensible son absence - donc en performant la disparition - par l'opacité d'un mot dont le référent est devenu insaisissable et incertain. 
6 De manière plus extensive, la disparition est un motif qui permet de penser la valeur productive et participative de la médiation. Si le statut ontologique d'un objet est incertain, le poids et l'importance des documents qui en attestent l'existence s'accroissent. L'agencement de documents ou d'archives devient le seul accès à la connaissance de l'objet, mais ces médiations ne constituent pas un lieu de passage vers celui-ci; elles en (re)produisent des membres ou des aspects et participent à son identité, tout en devenant elles-mêmes autres de par leur participation à l'agencement. La disparition est à la fois $l e$ produit et $c e$ qui produit l'émergence du système qui constitue l'objet, dans un même mouvement. Ainsi il ne s'agirait pas d'un vide ou d'une absence, mais du devenir d'un nouvel être au monde hypermédiat, c'est-à-dire un être au monde qui émerge des interactions entre médiations et du passage d'une médiation à l'autre dans un agencement que l'on peut considérer comme performatif. Ma position est en cela semblable à celle de Varsos et Wagner, qui stipulent que la disparition :

touche en effet de manière singulière [la question] des médias, du passage d'un médium à l'autre, des relations ou lacunes entre eux, pour autant que la production ou la reproduction de matériel ou de discours relevant de ce qui disparaît et de sa persistance, ou mémoire, soit entreprise par différents types de médias [...]. La quête à propos de ce qui disparaît nous confronte aux modes d'existence liminale qui semblent caractériser l'expérience contemporaine du réel, tissé par des liens et des creux intermédiaux. (Varsos et Wagner $2007: 13$ )

Ces liens inextricables entre la disparition et la médiation apparaissent comme un point de départ intéressant pour l'analyse de Dora Bruder, dont le premier quart est particulièrement riche pour penser le geste d'écriture d'un point de vue intermédial, à travers le prisme de la disparition. C'est notamment le statut originel de Dora Bruder comme référent extérieur originel qui sera ici mis à mal, ceci nécessitant de se détourner d'une logique de la représentation et de l'idée d'une médiation qui, telle une fenêtre, constituerait un lieu de passage vers le référent.

\section{Disparition et hypermédiateté}

8 Le présupposé qui sous-tend la présente approche est donc que la disparition de Dora Bruder, dans l'œuvre de Modiano, possède un statut singulier du fait que la jeune fille n'a effectivement pas d'identité qui précède sa disparition, contrairement aux autres personnages modianiens. C'est d'emblée la disparition qui la fait naître dans l'expérience de l'auteur. À ma connaissance, Dora Bruder serait ainsi le seul personnage essentiellement hypermédiat, c'est-à-dire le seul personnage qui n'a d'existence que par et dans la médiation et avec lequel le narrateur n'a, à aucun moment, un contact immédiat. Je m'inspire ici de la notion d'hypermediacy telle que proposée par Bolter et Grusin qui, à un endroit, la définissent comme caractérisant un espace hétérogène où « la représentation n'est pas conçue comme une fenêtre sur le monde, mais plutôt comme étant elle-même fenêtrée-avec des fenêtres qui s'ouvrent sur d'autres représentations ou d'autres médias. La logique de l'hypermédiateté multiplie les signes de médiation et, en ce sens, elle tente de reproduire le sensorium de l'expérience humaine ${ }^{2}$ »(Bolter et Grusin 1999: 34). Bien que les notions utilisées par Bolter et Grusin pour définir l'hypermédiateté (hypermediacy) - les médias, la représentation et les fenêtres - ne correspondent pas tout à fait au cadre épistémologique dans lequel je me situe, j'en retiens que les auteurs définissent une dynamique où la médiation n'ouvre pas vers un référent extérieur, mais vers d'autres médiations qui constituent 
finalement la seule existence de l'objet. L'adolescente est donc ontologiquement différente des autres protagonistes de Modiano, en ce sens qu'elle ne préexiste pas à l'avis de recherche et aux autres documents et archives qui lui seraient reliés : pour l'auteur, comme pour le lecteur, Dora Bruder est la somme de ces documents, sans en être extérieure.

De ce point de vue, la réelle consistance de Dora Bruder est directement liée à la matérialité des documents et archives que l'auteur-narrateur rassemble, et entremêlée aux réseaux de relations qui constituent les médialités ainsi dévoilées. Le geste d'écriture de Modiano, qui se positionne hors du genre narratif du récit de fiction, vient non pas représenter, mais se greffer et s'agencer à ce réseau dans le mouvement même où il l'expose, produisant ainsi un discours performatif sur les dispositifs qu'il déconstruit - incluant sa propre écriture - ou, du moins, dont il dévoile (ou occulte) certains rouages et modes de fonctionnement. C'est donc la relation entre le geste d'écriture de Modiano, sa valeur et ses relations évolutives et complexes avec Dora Bruder en tant qu'objet hypermédiat qui constituent la base du présent article. Le texte en tant que tel est ici conçu comme lieu à la fois de l'acte écrit et de ses processus de remédiation ${ }^{3}$. Ainsi, après avoir d'abord présenté en quoi Dora Bruder est l'espace d'une écriture-quête performative, je traiterai de l'écriture comme réponse au désir d'immédiateté, puis, dans un deuxième élan, de la transparence problématique d'un dispositif kafkaïen et d'une éthique de la remédiation telle qu'elle est mise en œuvre dans le premier quart du livre. La question de la mémoire, inévitable, traverse à la fois l'écriture de Modiano et la mienne. Une mémoire qui emprunte plusieurs formes, mais qui n'est surtout pas, contrairement à ce qui a souvent été répété, gardée par l'auteurnarrateur, qui trahirait son entreprise s'il l'immobilisait ainsi.

\section{« Douter de la littérature »}

\section{Voyage de noces : disparition comme événement et réponse de l'imaginaire}

10 Il est d'abord intéressant de comparer minimalement Dora Bruder à Voyage de noces pour voir en quoi la disparition, dans le livre de 1997, n'induit pas les mêmes rapports aux personnages et à l'écriture que dans la plupart des autres romans de l'auteur. Le récit fictif de Voyage de noces fait de la disparition un événement déclencheur de l'histoire de la jeune Ingrid, qui décide un soir de ne pas rentrer chez elle, puis de celle de Jean, narrateur emblématique des récits modianiens, qui performe lui-même une disparition de soi pour retracer des parties de la vie d'Ingrid, deux décennies plus tard. Si les quelques traces et documents trouvés sont reliés à la jeune femme, elle en est toujours absente et maintient une existence hors d'eux. Ingrid et Jean correspondent tous deux parfaitement au " personnage modianien [qui] disparaît parfois pour s'éviter de graves ennuis [ou qui] éprouve un désir très fort de disparaître à une certaine vie pour ensuite réapparaitre dans une autre » (Lecaudé $2007: 239$ ). La disparition est ici traitée comme un événement qui se produit dans le fil du récit, qui a un avant et un après, et qui, si elle détermine la trajectoire des personnages, ne remet pas en question leur existence en tant que telle, leur être au monde. Le personnage d'Ingrid, qui est en fait une stratégie de remise au monde de Dora, évolue en miroir de cette dernière : sa fugue et 
sa mort sont mises en récit, alors que ce sont précisément ces éléments qui demeurent inaccessibles et impénétrables, donc toujours en questionnement, chez Dora Bruder.

11 La démarche qui a mené à Voyage de noces traduit une dynamique qui correspond à la relation entre la disparition et l'imaginaire telle que la conçoit Santini (2007:35) : « [1]a disparition d'un corps solide ou d'une chose physique a toujours une suite, comme si elle était le point de départ d'une histoire. En fait, cette dynamique est très simple : tout élément qui disparaît fait place à l'imaginaire. " C'est effectivement par le biais de la fiction romanesque, impliquant une part nécessaire d'imaginaire, que Modiano tente d'abord de combler le manque que sa lecture de l'avis de recherche provoque, mais l'écriture de Voyage de noces ne permet pas suffisamment à Modiano de «se rapproch[er] d'elle [Dora Bruder], dans l'espace et le temps» (DB: 54). Ainsi la disparition considérée comme une absence résultant et engendrant désir et imaginaire a mené vers un roman qui s'inscrit en continuité avec le restant de l'œuvre de l'auteur, mais la création de ce monde fictionnel n'a pas permis le contact avec la jeune fugueuse qu'il recherchait: la disparition de Dora Bruder (que Modiano fait également disparaître de Voyage de noces, d'une certaine manière, en l'en excluant) a conservé un effet de négation et de vide, maintenant effectif le manque qu'une mise en récit n'aura pas pu combler, malgré le fait que « l'écriture du roman devait avoir en quelque sorte une dimension performative" (Sheringham 2009: 259) en permettant à l'auteur de « continuer à concentrer [s] on attention sur Dora Bruder» $(D B: 53)$.

\section{Dora Bruder : performativité et procès de l'écriture comme quête}

12 Entre l'écriture de Voyage de noces et celle de Dora Bruder survient une importante remise en question de l'écrivain, dont il fait part en 1994 dans un article de Libération, disant qu'il s'est mis à «douter de la littérature ${ }^{4}$ ». Sans verser dans l'analyse psychologique, il semble que le fait que l'écrivain, qui s'inscrit lui-même dans Dora Bruder, ait commencé à « remettre en cause le statut de la fiction romanesque [et se poser] la question du rôle du roman» (Amar 2011: 351) peu de temps avant la publication du livre soit éclairant pour penser au geste d'écriture, qui se veut finalement un acte de médiation hors (ou détourné) de la médialité de la littérature et des «normes littéraires préconçues » (Ibid.). S’il était déjà possible de reconnaître une part de performativité au roman Voyage de noces, il semble que cette performativité soit exacerbée dans Dora Bruder, au point peut-être d'en faire un performatif en soi, qui « se référ[e] à une réalité qu'il constitue lui-même » (Benveniste 1966 : 274). Cette réalité correspond à la recherche en tant que telle : la quête de Dora Bruder, qui ne se fait pas seulement dans les établissements où se trouvent les archives, mais aussi dans le geste d'écriture qui organise, découpe, agence et fouille ainsi les documents en les exposant et réfléchit sur lui-même, puis la quête de la légitimation d'une écriture capable de déjouer ces normes préconçues de la fiction romanesque.

Ce qui est mis en œuvre est en fait un geste d'écriture qui ouvre sans cesse vers l'extérieur et qui expose son propre devenir, traduisant une impossibilité de faire œuvre en plaçant le travail de l'écriture dans une logique de la performance, du côté des «processus dynamiques en temps réel plutôt que des objets statiques ou des représentations ${ }^{5} »$ (Salter 2010: XXIII). Modiano détourne ainsi l'opposition entre l'œuvre et le document, selon laquelle «l'œuvre suppose une autonomie, une autosuffisance, relativisée par le contexte, le document n'est jamais suffisant ni fermé 
sur lui-même " (Chevrier et Roussin cités dans Zenetti 2012: 31). Dora Bruder vient plutôt s'agencer avec les documents qui y sont remédiés en mettant en évidence sa propre impossibilité à tout révéler, son incomplétude et, surtout, son aspect processuel et inachevé. Modiano ne se présente donc pas comme un "gardien de la mémoire " (Schulte Nordholt $2011: 524$ ), mais comme l'acteur d'une quête et d'une construction de la mémoire, qui ne doivent pas avoir de finalité. Le livre est, en même temps que la quête elle-même, l'agenda d'une recherche en devenir, qui se manifeste sous le mode du " un jour je ", réitéré dans les premières pages du texte ("Un jour, j'irai à Sevran » $[D B: 19]$ « Un jour, j'irai. Mais j'hésite. » [DB: 14] «Un jour, je retournerai à Vienne » [DB : 22]), ainsi qu'un lieu de questionnements, tant sur les données recherchées que, de façon peut-être plus importante, sur la méta-question que ces questionnements amènent : « comment savoir ?» (DB: 22).

L'écriture entretient avec la disparition une relation différente de celle qui est normalement à l'œuvre dans les romans de fiction modianiens. Il n'y a pas de récit possible pour la disparition de Dora, qui n'a pas plus de fin qu'elle n'a d'origine, tout comme il n'y a pas d'origine à la quête de Modiano, qui réécrit sa mémoire pour être déjà, en 1965, «sur la trace de Dora Bruder et de ses parents. Ils étaient déjà là, en filigrane » (DB: 11). Tous deux, Dora Bruder et Dora Bruder, sont déjà entremêlés dans un agencement intermédial dont ils sont inséparables. Si «ce qui disparaît persiste dans son absence, de manière irrésolue, certes, mais incitant aussi l'émergence de nouvelles modalités de présence ou de vie » (Varsos et Wagner 2007 : 12), il semble que ce soit justement ces nouvelles modalités qui sont problématisées et traitées de façon différente dans l'écriture hors du récit de fiction que Modiano a mise en œuvre. Dora Bruder érige une sorte de portrait cubiste d'un personnage hypermédiat dans un milieu textuel qui répond lui-même à cette hypermédiateté, dans le mouvement même où il la construit.

\section{D'abord, être écrivain}

\section{L'écriture en relief}

Il est utile de préciser que pour les théoriciens des médias Bolter et Grusin, l'hypermédiateté est une logique indissociable de l'immédiateté, qui en est le contrepoint et non le contraire. Ces deux auteurs expliquent l'une des modalités de leur interrelation, qui implique une sorte de circularité, ainsi: "Dans toutes ses manifestations, l'hypermédiateté nous rend conscients du medium ou du média et (de façon plus ou moins subtile) nous rappelle notre désir d'immédiateté ${ }^{6}$ " (Bolter et Grusin 1999: 35). Cette logique contrapuntique s'avère productive pour analyser les mouvements et motivations complexes de l'écriture dans l'ouverture de Dora Bruder.

Rappelons que le texte s'ouvre sur la mention du Paris-Soir de 1941 et sur la citation de l'avis de recherche, qui mentionne le nom de Dora Bruder. La dynamique intermédiale, très prégnante par endroits dans le livre, n'est par contre plus manifestée dans les quelques pages qui suivent. Ces sections sont écrites dans un régime assez typique des narrations modianiennes, bien que les temps et lieux, ainsi que les liens causaux, soient peut-être davantage brouillés que dans ses autres romans. La surimpression des époques, procédé courant chez Modiano qui reprend parfois des formules très proches 
d'un livre à l'autre ${ }^{7}$, est complexifiée dans Dora Bruder, où le brouillage se fait cette fois entre 1942, 1965, 1988 et 1996-1997.

Il semble toutefois qu'il y ait une importance accrue accordée à l'acte d'écriture, dans ces premières pages de Dora Bruder, qui mettent en relief le geste de l'auteur en train d'écrire. Il y a d'abord un mouvement autoréflexif installé dès les premières lignes, mouvement mettant en évidence le potentiel d'expression de l'écrivain par la remédiation - isolée mais initiale - du Paris-Soir et de l'avis de recherche, avec lesquels une différence est soulignée par la disposition des paragraphes et par l'utilisation de guillemets, "explicitly pointing to a medial difference, to its own mediality" (Rajewsky 2010 : 58). Cette dernière citation d'Irina O. Rajewsky, qui traite des relations intermédiales mises en œuvre dans un seul produit matériellement présent tel que le fait Dora Bruder, va également dans le sens des propos de Walter Moser (2007 : 80), selon qui « la médialité d'un art [...] devient nécessairement apparente quand deux médias différents entrent en jeu et interfèrent ». Cela contribue ainsi à mettre en lumière le paradoxe que je repère dans Dora Bruder (et dans d'autres œuvres qui présentent une dynamique semblable) : l'agencement du texte de Modiano avec ces autres médiations qui l'informent, qu'elles soient textuelles ou non, renforce en fait la prégnance de l'acte d'écriture de l'auteur et, ainsi, l'aspect performantiel (qui s'ajoute à l'aspect performatif déjà soulevé, mais qui ne s'y confond pas) de l'écriture et de la médialité qu'elle affiche.

\section{Performance et identité}

Cette performance du geste d'écriture se confond plutôt avec la performance de l'identité de l'écrivain, qui écrit pour être. En 1965, «je n'étais rien, je me confondais avec ce crépuscule, ces rues » (DB: 8), écrit Modiano, faisant ici référence à une période qui précède de deux ans la parution de son premier roman. Hors de l'écriture, l'auteur n'existe pas. Il y a ainsi un déplacement de la subjectivité et une thématisation du devenir-écrivain qui ne se confond plus avec le décor qu'il habite, mais avec son écriture et qui, de cette façon, se manifeste lui-même dans sa propre création. Ainsi l'auteur-narrateur se construit, au fil de son texte, et il se présente comme étant déjà à la recherche de Dora Bruder, cet objet hypermédiat tel que je l'ai déjà défini - même avant que l'avis de recherche ne lui confère une réelle part d'existence pour Modiano en se positionnant lui-même dans l'écriture qu'il utilise pour rechercher et pour reconstruire Dora. Il crée une mémoire et une expérience qui ne sont pas préexistantes ou extérieures à l'écriture et qui pourraient ainsi contribuer à le rapprocher de Dora dans cette hypermédiateté, dans une logique de la création et non de l'identification. Plutôt qu'entre les années et les lieux, le vrai brouillage se situerait donc entre la mémoire, l'expérience et l'écriture, qui se construisent dans un même mouvement. Comme Georges Perec le soulignait, « toute expérience ouvre à la littérature et toute littérature à l'expérience » (cité dans Jurt 2007 : 91) et j'ajouterais, dans le cas présent, à la mémoire, qui n'en est pas éloignée : "Si le simple témoignage, les documents, la récolte des faits ont une valeur pour les survivants et bien sûr pour les historiens, ce n'est que la parole, la force créatrice de l'écrivain qui saura faire revivre les faits pour ceux qui n'ont pas fait personnellement cette expérience » (ibid.).

L'idée de performance implique un important paradoxe de l'immédiateté, dans le temps et l'espace, d'un acte de médiation. C'est ici que la phrase de Bolter et Grusin que 
j'ai citée plus tôt prend son sens, pour supporter l'hypothèse selon laquelle la mise en évidence de l'écriture, qui apparaît comme une performance de soi, met en lumière une double dynamique: si l'écriture contribue à faire de Dora un personnage essentiellement hypermédiat, elle fait aussi de l'auteur un être qui est par et dans son acte, et rappelle donc son désir d'immédiateté, le désir qu'il a de pouvoir provoquer un contact direct avec Dora Bruder, dont il ne peut se rapprocher que par un acte de médiation supplémentaire.

\section{S'écrire une mémoire}

C'est donc cette écriture de la mémoire (et en même temps, la mémoire par l'écriture) qui apparaît comme étant l'avenue privilégiée pour composer de prime abord avec le désir d'immédiateté qui traverse l'œuvre. L'auteur-narrateur, à partir des éléments contenus dans l'avis de recherche (le lieu, les noms, les dates) aligne des paragraphes qui forment autant de souvenirs, dans une forme qui n'est pas étrangère aux « Je me souviens » de Georges Perec (1978), bien que cette expression ne soit explicitement utilisée qu'une fois ( $D B: 8$ ). Au bout d'une première série de souvenirs qui n'appartiennent qu'au « je » de l'auteur, le chapitre se clôt sur l'appropriation de l'avis de recherche par celui qui écrit, une dissolution du « Adresser toutes indications à M. et Mme Bruder, 41 boulevard Ornano, Paris » (DB:9), sans guillemets cette fois, dans son écriture. Si les perspectives se brouillent pour l'auteur avec les années, elles sont plutôt brouillées, pour le lecteur, par ce procédé de remédiation qui dissout les frontières entre l'écriture du livre et celle du journal, dont les données semblent tout à coup issues de la résurgence des souvenirs de l'auteur.

21 Ce procédé est rendu plus explicite aux pages suivantes, où l'on reconnait qu'il écrit ses souvenirs pour faire en sorte que Dora et ses parents se manifestent déjà, quelque part, dans sa mémoire. Il écrit: "Peut-être, sans que j'en éprouve encore une claire conscience, étais-je sur la trace de Dora Bruder et de ses parents. Ils étaient là, déjà, en filigrane. / J'essaye de trouver des indices, les plus lointains dans le temps " (DB: 10-11). Ce qu'il "essaye " consiste donc en un détournement du statut de l'avis de recherche (et, en même temps, la disparition) comme événement déclencheur de sa quête et une négation - ou du moins une réduction - de la distance entre ce qu'il recherche et lui-même, en les faisant exister, déjà, dans sa propre mémoire que l'écriture produit.

Cela fait d'ailleurs écho au « don de voyance » (DB:52) qui se voit plus tard attribué aux romanciers, don qui "peut sans doute provoquer à la longue de brèves intuitions "concernant des événements passés ou futurs" » (DB : 53). Le romancier est ici celui qui peut saisir et reconfigurer des réalités d'autres temps, faisant passer les intuitions dans le régime de la vérité par et dans son geste d'écriture. Ce geste d'écriture dans Dora Bruder apparaît comme modalité de construction d'une mémoire différente de l'écrivain qui performe et construit sa singularité dans ce geste, se rapprochant ainsi momentanément de la jeune fugueuse dans un phénomène patent d'hypermédiateté dans ce qu'elle implique comme reconfiguration de l'immédiateté. 


\section{Devant le dispositif} rapproche de l'adolescente en diffusant l'existence de cette dernière dans sa propre mémoire, est suivie par un chapitre où la quête prend un aspect beaucoup plus concret, avec des données plus précises sur les démarches de recherche entreprises et sur les données en tant que telles. Après avoir livré «la date exacte de sa naissance: le 25 février 1926 [et] le lieu de cette naissance : Paris, XII ${ }^{e}$ arrondissement» (DB : 14), le narrateur fait le récit des étapes par lesquelles il a dû passer pour avoir accès à une copie intégrale de l'acte de naissance de Dora. Comme Nicolas Xanthos l'a déjà souligné (2011: 223), certains passages de ce chapitre rappellent parfois «avec insistance le Devant la Loi de Kafka", dont l'un des deux seuls personnages est une sentinelle qui bloque l'accès à la Loi. Si la critique modianienne s'est généralement concentrée sur Dora Bruder en tant qu'elle est "plongée dans l'absence » (Schulte Nordholt 2011 : 534), je voudrais ici exposer la complexité de ce qui lui confère en fait encore une présence au monde, soit les archives qui conservent sa plus petite part d'existence - son nom mais qui, en même temps, la met en péril. Selon Dominique Viart (2010:67), « le livre ne se contente pas de dresser un "monument" à Dora, mais entreprend de rendre tangible son absence, d'en faire l'épreuve sensible ». Cette affirmation est juste, mais il est intéressant de la poursuivre en insistant sur un autre versant : le livre met en même temps en évidence le poids, d'une part très léger, et de l'autre, très lourd, de l'hypermédiateté tributaire de la disparition. Du léger petit acte de naissance enfoui sous le lourd dispositif kafkaïen, dont la mise en place et le maintien sont justement déterminés par la somme de ces toutes petites traces de mémoire qui en font partie.

\section{De quelques transparences}

Après les notions d'immédiateté et d'hypermédiateté dont j'ai précédemment traité, il semble que ce soit la dynamique entre opacité et transparence qui puisse être problématisée à partir de l'émergence, dans l'écriture, d'un dispositif explicitement présenté comme « croisement des relations de pouvoir et de savoir » (Agamben 2007 : 11). Si ces deux dialectiques ont parfois été présentées comme synonymes, depuis Remediation de Bolter et Grusin (1999), faisant parfois de "transparence / opacité » l'équivalent français des notions de "immediacy/hypermediacy", force est de constater qu'elles soulèvent des enjeux très différents lorsque l'on sort du paradigme de la représentation visuelle. Les notions de transparence et d'opacité engendrent et impliquent effectivement des dynamiques qui se distinguent des deux autres termes, la transparence étant d'ailleurs un concept complexe qui peut avoir plusieurs acceptions ${ }^{8}$. Chez Bolter et Grusin, l'immediacy est définie par rapport à une sorte d'effacement du média : c'est "l'idée qu'un medium puisse s'effacer de lui-même et laisser le spectateur en présence des objets représentés, pour que ce dernier atteigne une connaissance directe des objets ${ }^{9}$ " (Bolter et Grusin 1999: 48). On pense ici à l'idéal de la réalité virtuelle, où le dispositif de médiation se ferait complètement transparent (jusqu'à n'être plus perçu) pour donner l'impression à l'usager qu'il est réellement en contact avec ce qui est représenté. S'il y a effectivement un idéal de la transparence de la médiation dans le cas de la réalité virtuelle, la notion de transparence peut également concerner d'autres phénomènes qui ne relèvent pas uniquement de l'effacement du 
média ou du contact direct avec le représenté. La tendance vers la naturalisation de ce qui demeure pourtant bien tangible, par exemple, en est un: "En introduisant des objets graphiques dans le système de représentation, les designers ont cru qu'ils rendaient l'interface "transparent", et donc, plus "naturel" "10 (ibid. : 32). Ce qui est en jeu, dans le chapitre de Dora Bruder, concerne encore une autre forme de transparence qui, pour poursuivre les comparaisons avec des artefacts, correspondrait à l'utilisation d'une enveloppe ou d'une coquille de plastique transparent exposant le mécanisme de fonctionnement, parfois hautement sophistiqué et complexe, de l'objet en question. Avec Buydens (2004:54-55), on peut dire que « le produit transparent est un produit qui s'ouvre et se révèle, contre l'artifice de l'enveloppe qui dissimule». L'opacité, en contrepoint, serait ce qui bloque ce dévoilement.

\section{Bloquer pour fonctionner}

Lorsque l'auteur-narrateur se rend à la mairie du XII ${ }^{\mathrm{e}}$ arrondissement, service de l'état civil, puis au palais de justice où il se bute à quelques "plantons", à une queue de touristes, à des gardiens, il croise des représentants de la loi à qui il n'ose pas "demander par où on accèd[e] à l'escalier 5 » (DB:17) qu'il cherche et il apprend qu'il doit plutôt « écrire à $M$. le procureur de la République, parquet de grande instance de Paris, 14 quai des Orfèvres, $3^{e}$ section $B$ » (DB: 18) pour finalement recevoir l'acte de naissance en question, trois semaines plus tard, par la poste. En retraçant ce chemin, dans une écriture qui est, de plus, bloquée par des remédiations improductives comme celle d'un formulaire qui se dresse au lieu d'une parole directe et qui amène, de toute façon, une réponse négative - l'auteur performe une transparence de sa démarche d'écriture, qui n'est pas celle d'un réalisme qui tendrait à rendre les personnages et lieux plus réels pour le lecteur, mais une transparence éthique, qui met en avant une forme d'honnêteté qui ne déleste pas les informations recueillies du poids de leur médialité et qui ne dissimule pas le chemin qui a mené aux données livrées par l'acte de naissance (lui aussi recopié entre guillemets). Ce faisant, tel un ordinateur ou un téléphone transparent, il affiche en même temps la complexité du système qui a informé son écriture-quête. Un système qui apparaît dans ses ramifications, dans ses blocages, bref, dans son opacité constitutive.

Ces blocages traduisent en fait toute l'efficacité du dispositif qui semble dressé autour de l'acte de naissance de Dora. Les propos de Galloway (2012:25) expliquent bien cette logique : "plus un dispositif devient intuitif, plus il risque de ne plus être reconnu comme un média, apparaissant aussi naturel que l'air ou aussi commun que la poussière $^{11}$ ». Galloway poursuit en citant Michel Serres qui, lui, parle des parasites essentiels au maintien de la relation de communication: "Les systèmes marchent parce qu'ils ne marchent pas. Le non-fonctionnement demeure essentiel pour le fonctionnement [...] [L]a relation est la non-relation. Et c'est cela, le parasite " (Serres 1980 : 107). Chaque gardien qui performe son rôle de planton en ralentissant la progression de l'auteur, qui lui fournit des indications imprécises, qui le dirige vers une autre instance, assure en fait le "bon» fonctionnement et la pérennité relative des structures qui régulent les relations de pouvoir et de savoir qui, si elles accentuent les mécanismes de blocage qui assurent leur prégnance, risquent de faire disparaitre tout à fait les archives, comme "ces sentinelles de l'oubli chargées de garder un secret honteux, et d'interdire à ceux qui le voulaient de retrouver la moindre trace de 
l'existence de quelqu'un » (DB: 16). En même temps, cette résistance des plantons et de l'administration est précisément ce qui augmente le poids de l'existence de Dora, qui n'est pas plongée dans l'absence, mais dans la réalité des documents, des communications et des institutions se concentrant autour d'un acte de naissance qui acquiert une dimension presque auratique.

\section{Une remédiation éthique}

Ces blocages, ce sont aussi ceux du récit biographique. Les informations livrées par l'acte de naissance auraient pu faire l'objet d'une narration installant les lieux, les personnages, la situation initiale - littéralement - de la vie de Dora, mais ce procédé aurait gommé et amoindri une importante part de l'existence (hypermédiate) de la jeune fille. Il y a donc une forte dimension éthique qui est reliée à cette remédiation de l'acte de naissance qui, avant d'être finalement livré entre guillemets, et au lieu d'être oublié au fond des archives, contribue à exposer les rouages du dispositif dans lequel le document est finalement mis en mouvement et dont le poids et la valeur sont augmentés par ce dispositif, qui apparaît en même temps comme dépendant des minuscules éléments devant lesquels il fait bloc. L'être au monde de Dora et du dispositif apparaissent tous les deux, à ce moment, comme tributaires de la recherche de l'acte de naissance. Ce passage montre également en quoi il est problématique de considérer Modiano comme un gardien de la mémoire : les gardiens, dans Dora Bruder et dans l'œuvre de cet auteur en général, sont au contraire ceux qui menacent la mémoire, ce sont ceux qui peuvent la détruire ou la cacher plutôt que la transmettre.

Une autre forme d'éthique de la remédiation se dégageant des premières pages de Dora Bruder est manifestée dans la section suivante, qui commence par la dissolution de quelques mots de l'acte de naissance dans l'énonciation de Modiano, sans guillemets, et avec le passage des nombres en chiffres arabes plutôt qu'en lettres : « Ernest Bruder. Né à Vienne, Autriche, le 21 mai 1899 " (DB : 21). Dans cette section, l'auteur « essaye de reconstituer le périple d'Ernest Bruder " $(D B: 23)$ à partir de ses date et lieu de naissance et de quelques données sur le contexte social de l'époque. L'auteur sait " qu'Ernest Bruder a été " $2^{\mathrm{e}}$ classe, légionnaire français" » (DB:23) et c'est à partir de cette autre information, contenue sur une seconde petite fiche, qu'il tente de proposer un chemin qui aurait pu mener le père de Dora vers Paris, où il a marié la mère de Dora, à 25 ans.

Ce dernier document est en fait « une petite fiche parmi des milliers d'autres établies [...] pour organiser les rafles de l'Occupation et qui traînaient jusqu'à ce jour au ministère des Anciens Combattants » (DB:23). Cette fois, contrairement à ce qui est mis en œuvre dans le cas de l'acte de naissance de Dora, la fiche en question n'est pas citée dans son entièreté. L'auteur se limite à ce que j'ai moi-même reproduit au paragraphe précédent, puis ajoute, quelques pages plus loin : "La petite fiche d'Ernest Bruder, " 2 e classe légionnaire français", indique aussi "mutilé de guerre $100 \%$ ” (DB: 25). Les guillemets sont toujours conservés; le contenu de la fiche ne passe jamais dans l'énonciation de l'auteur, qui ne se l'approprie donc à aucun moment. Ce petit document est lui-même mutilé, fragmenté, disséminé dans les pages et maintenu dans une sorte de distance par rapport à l'acte d'écriture. Certaines données de cette fiche resurgissent effectivement à quelques endroits, mais ce n'est que beaucoup plus tard qu'elle apparait, toujours entre guillemets, dans toute son horreur, dans un chapitre 
qui commence cette fois par «Ernest Bruder a été arrêté le 19 mars 1942, ou, plus exactement, interné au camp de Drancy ce jour-là » (DB : 81) :

« Bruder Ernest

21.5.99 - Vienne

$\mathrm{n}^{\circ}$ de dossier juif : 49091

Profession : Sans

Mutilé de guerre $100 \% .2^{\mathrm{e}}$ classe légionnaire

français gazé ; tuberculose pulmonaire.

Casier central E56404»

Plus bas la fiche porte une inscription au tampon : RECHERCHÉ, suivie de cette note au

crayon : «Se trouve au camp de Drancy. » (DB: 81)

30

ières années de vie du père de Dora preuve de son assassinat. $\mathrm{Si}$, au début du roman, un acte de naissance peut apparaître dans son entièreté, avec la typologie et les normes d'écriture qui lui sont propres, il semble qu'une fiche établie pour mieux faire disparaître un individu ne puisse pas recevoir le même traitement. La charge morbide de ces documents est résumée: «Ceux-là mêmes qui sont chargés de vous chercher et de vous retrouver établissent des fiches pour mieux vous faire disparaître ensuite - définitivement » (DB:82).

Il y a donc, dans ce chapitre qui fait partie des sections inaugurales du livre érigées en lieux de création plutôt que déterminées par les mécanismes de destruction, une manipulation et un détournement de l'un des documents les plus puissants dans ses effets négatifs qui s'agence à l'écriture de Modiano. Comme pour en renverser la charge, il l'utilise dans un chapitre dédié au devenir du père de Dora, à son émergence dans le livre ainsi qu'à son destin probable depuis sa propre naissance jusqu'à son mariage, et se sert donc de la fiche - presque toujours accompagnée de l'épithète « petite» qui contraste avec la lourdeur des informations qui y sont inscrites - pour lui créer un nouveau trajet de vie qui, bien que spéculatif, correspond à un mouvement contraire à celui qui aurait un jour été relié à cette fiche. Ce n'est donc pas, dans cette section du livre, Ernest Bruder qui disparaît dans la fiche, mais l'effectivité de cette petite fiche qui disparaît en étant détournée, rompue, occultée et mise à distance par l'écriture de Modiano. Dans le cas présent, le procédé de remédiation qui consiste à inclure des parties de la fiche d'Ernest dans Dora Bruder apparaît comme une démarche de distanciation nécessaire, un refus de dissoudre l'acte d'écriture de la fiche dans l'acte d'écriture de l'écrivain. Les guillemets demeurent la première et la plus efficace façon de dire «ce n'est pas moi qui ai d'abord écrit cela. Je n'en suis pas (le) responsable ».

\section{Conclusion}

Cette éthique de la remédiation est effectivement de première importance si l'on accepte le présupposé selon lequel les «personnages » de Dora Bruder n'ont pas une existence idéelle qui soit extérieure aux médiations qui continuent de leur conférer une part d'être au monde. La manipulation de ces modes d'existence serait ainsi liée à une manipulation de leur identité en tant que telle. Dans cette perspective, les remédiations, dans Dora Bruder, ne servent pas l'unique but d'accréditer l'authenticité de la quête, mais celui de faire en sorte que leur part d'existence soit "alourdie ", 
comme si le poids du livre qui les remet au monde correspondait à la somme de la masse des médiations qui y prennent forme.

Dans cette logique, Dora Bruder oppose une résistance au récit biographique et propose une alternative à l'idée de disparition conçue comme événement final: Dora Bruder - le livre - est lui-même le point de départ et le lieu d'une conflictuelle existence hypermédiate pour Dora, pour ses parents, et pour les quelques autres individus qui sont nommés à travers les pages (cette existence transcendant d'ailleurs ce que l'on entend conventionnellement par « individu»). Comme le soulignent Varsos et Wagner (2007: 12), la disparition suppose " qu'il y ait suspension ou interruption plutôt que clôture ou accomplissement et qu'il manque la certitude de la fin qui aurait permis d'identifier et articuler une finalité » et elle est ainsi ouverte dans le temps, mais j'ajouterais qu'elle implique aussi un éclatement de ce qui disparaît, qui se manifeste dans une multitude de relations qui viennent reconfigurer les limites de l'individu. La relation entre Dora Bruder et le dispositif kafkaïen est un exemple de cet éclatement qui excède les frontières de la pure biographie.

La mémoire de Dora Bruder est davantage à transmettre, à partager et à compléter plutôt qu'à garder ou conserver. Dora Bruder est tourné vers une ouverture et un extérieur ; c'est une œuvre qui performe son devenir et son incomplétude, à partir de l'intériorité de l'écrivain parfois, mais jamais à partir de l'intériorité de Dora qui serait, de toute façon, fantasmée. L'adolescente conservera à jamais son " pauvre et précieux secret » (DB : 145). Il s'agit bien d'un portrait cubiste qui ne prétend pas dire qui était Dora, mais qui, après avoir accepté qu'elle est un être hypermédiat, s'applique à la remettre en mouvement dans un milieu intermédial mû par un désir d'immédiateté. Le rapport à la réalité de Dora Bruder n'est donc pas seulement celui d'un récit qui se base sur des faits réels, mais plutôt celui d'une œuvre qui rend explicite sa participation à la création de la réalité.

\section{BIBLIOGRAPHIE}

Agamben, Giorgio, 2007, Qu'est-ce qu'un dispositif?, Paris, Payot Rivages.

Amar, Ruth, 2011, «Le ton de Patrick Modiano : du roman ironique au roman affectif », Analyses, vol. 6, $\mathrm{n}^{\circ} 1$ (hiver), p. 343-361.

Benveniste, Émile, 1966, Problèmes de linguistique générale, t. I, Paris, Gallimard.

Bolter, Jay David et Grusin, Richard, 1999, Remediation. Understanding New Media, Cambridge, MIT Press.

Buydens, Mireille, 2004, « La transparence : obsession et métamorphose », Intermédialités, $\mathrm{n}^{\circ}$ 3, p. 51-77.

Galloway, Alexander R., 2012, The Interface Effect, Maiden, Polity Press.

Jurt, Joseph, 2007, « La mémoire de la Shoah : Dora Bruder », dans J. E. Flower (dir.), Patrick

Modiano, Amsterdam, New York, Rodopi, p. 89-108. 
Lecaudé, Jean-Marc, 2007, «Patrick Modiano : le narrateur et sa disparition ou qu'y a-t-il derrière le miroir ? ", dans J. E. Flower (dir.), Patrick Modiano, Amsterdam, New York, Rodopi, p. 239-256.

Moser, Walter, 2007, «L'interartialité : pour une archéologie de l'intermédialité », dans M. Froger et J. E. Müller (dir.), Intermédialité et socialité. Histoire et géographie d'un concept, Münster, Nodus Publikationen, p. 69-92.

Rajewsky, Irina O., 2010, « Border Talks: The Problematic Status of Media Borders in the Current Debate about Intermediality », dans L. Elleström et J. Bruhn (dir.), Media Borders, Multimodality and Intermediality, New York, Palgrave Macmillan.

Salter, Chris, 2010, Entangled, Cambridge, MIT Press.

Santini, Sylvano, 2007, « La perception du mouvement entre disparition et apparition : réminiscence mallarméenne de l'intermédialité », Intermédialités, n 10, p. 33-54.

Serres, Michel, 1980, Le Parasite, Paris, Grasset et Fasquelle.

Sheringham, Michaël, 2009, « Le dispositif Voyage de noces - Dora Bruder », dans R.-Y. Roche (dir.), Lectures de Modiano, Nantes, Éditions Céciles Defaut, p. 243-266.

Schulte Nordholt, Annelies, 2011, «Photographie et image en prose dans Dora Bruder de Patrick Modiano », Neophilologus, [En ligne], p. 523-540.

DOI : $10.1007 / \mathrm{s} 11061-011-9288-7$

Varsos, Georges et Wagner, Valeria, 2007, « Disparaître à présent. Introduction », Intermédialités, $\mathrm{n}^{\circ} 10$, p. 9-16.

Viart, Dominique, 2010, « L'impossible narration de l'histoire », dans A.-Y. Julien (dir.), Modiano ou les intermittences de la mémoire, Paris, Hermann.

Xanthos, Nicolas, 2011, « Sentinelles de l'oubli et reflets furtifs. Permanence, rupture et régimes d'historicité dans Dora Bruder », Orbis Litterarum, vol. 66, n 3, p. 215-237.

Zenetti, Marie-Jeanne, 2012, « Prélèvement / déplacement : le document au lieu de l'œuvre », Littérature, $\mathrm{n}^{\circ} 166$, p. 26-39.

\section{Euvres littéraires citées}

Modiano, Patrick, 1990, Voyage de noces, Paris, Gallimard.

Modiano, Patrick, 1993, Chien de printemps, Paris, Seuil.

Modiano, Patrick, 1997, Dora Bruder, Paris, Gallimard.

Perec, Georges, 1978, Je me souviens, Paris, Hachette.

\section{NOTES}

1. Les renvois à cette édition seront notés $(D B)$, suivi du numéro de page, dans le corps du texte.

2. « representation is conceived of not as a window on to the world, but rather as windowed itself - with windows that open on to other representation or other media. The logic of hypermediacy multiplies the signs of mediation and in this way tries to reproduce the rich sensorium of human experience " (ma traduction).

3. C'est-à-dire de processus d'inclusion d'autres médiations par et dans (le milieu de) l'écriture.

4. Patrick Modiano, « Avec Klarsfeld, contre l'oubli », Libération, 2 novembre 1994. 
5. « real-time, dynamic processes over static objects or representations » (ma traduction).

6. «In every manifestation, hypermediacy makes us aware of the medium or media and (in sometimes subtle and sometimes obvious ways) reminds us of our desire for immediacy " (ma traduction).

7. Notons la proximité entre «La distance des années avait brouillé les perspectives » de Chien de printemps ( $C P: 91)$, où « le mois d'avril se fondait par un phénomène de surimpression avec celui d'avril 1964 » (CP:18) et cette même impression qui se dessine dans Dora Bruder : « avec le recul des années, les perspectives se brouillent pour moi, les hivers se mêlent l'un à l'autre » (DB:10).

8. Pour avoir un bon aperçu de la complexité de ce sujet, voir l'article de Mireille Buydens, «La transparence : obsession et métamorphose » (2004).

9. «the notion that a medium could erase itself and leave the viewer in the presence of the objects represented, so that he could know the objects directly » (ma traduction).

10. «By introducing graphical objects into the representation scheme, designers believed that they were making the interface "transparent" and therefore more "natural" " (ma traduction).

11. "the more intuitive a device becomes, the more it risks falling out of media altogether, becoming as naturalized as air or as common as dirt » (ma traduction).

\section{RÉSUMÉS}

La disparition est-elle un événement? Une fin? Une figure? Chez l'écrivain français Patrick Modiano, il semble qu'elle prenne plutôt la forme d'une invitation, d'un point de départ pour une écriture-quête se faufilant dans les mailles de la médiation. Le lien inextricable entre la disparition et la médiation est effectivement à la base de cette analyse de Dora Bruder, roman dont l'ouverture est particulièrement riche pour penser le geste d'écriture d'un point de vue intermédial. Après avoir présenté Dora Bruder comme l'espace d'une écriture-quête performative qui répond à un désir d'immédiateté, j'exposerai certains dispositifs traduisant une éthique de la remédiation. La question de la mémoire, inévitable, traverse à la fois l'écriture de Modiano et la mienne. Une mémoire protéiforme et dynamisée par l'auteur-narrateur.

What exactly do we mean by "disappearance"? Is it an event? A figure? The end of an existence? For Patrick Modiano, disapearance is rather a starting point for an act of writing that slips through a sum of mediations. The inextricable link between disappearance and (re)mediation is in fact the basis upon which I build my analysis of Dora Bruder's opening pages from an intermedial stance. Ethical remediation and performativity will be the core concepts to analyse Modiano's writing through the lens of disappearance.

\section{INDEX}

Keywords : Dora Bruder, Patrick Modiano, intermediality, disappearance, remediation

Mots-clés : Dora Bruder, Patrick Modiano, intermédialité, disparition, remédiation 
AUTEUR

ÉLISABETH ROUTHIER

Université de Montréal, Département de littérature comparée, Membre de la Chaire de recherche sur les Écritures numériques 